\title{
Cost Analysis of New Antibiotics to Treat Multidrug- Resistant Bacterial Infections: Mind the Gap
}

Dafna Yahav (D) - Daniel Shepshelovich · Noam Tau

Received: December 17, 2020 / Accepted: January 30, 2021 / Published online: February 13, 2021

(c) The Author(s) 2021

\begin{abstract}
Introduction: Guidelines for treatment of multidrug-resistant (MDR) bacteria rely on newly approved antibiotics, with limited evidence of their effectiveness for treating these infections. Data regarding cost of such an approach are lacking. We aimed to evaluate estimated cost of using newly approved antibiotic drugs compared to older antibiotics for the treatment of difficult-to-treat pathogens.

Methods: MDR bacteria of interest included those defined by the World Health Organization as critical or of high priority for research. Old and newly approved antibiotics for these bacteria, defined as approved before or after
\end{abstract}

Supplementary Information The online version contains supplementary material available at https:// doi.org/10.1007/s40121-021-00412-y.

D. Yahav $(\square)$

Infectious Diseases Unit, Rabin Medical Center, Beilinson Hospital, Petah Tikva, Israel

e-mail: dafna.yahav@gmail.com

D. Yahav · D. Shepshelovich · N. Tau

Sackler School of Medicine, Tel-Aviv University, Tel

Aviv, Israel

D. Shepshelovich

Medicine T, Sourasky Medical Center, Tel Aviv, Israel

N. Tau

Department of Diagnostic Imaging, Sheba Medical

Center, Ramat Gan, Israel
January 2010, respectively, were evaluated for treatment cost and for 14-day treatment course. Estimated annual costs were calculated based on the Centers for Disease Control and Prevention's' report on MDR bacteria prevalence in US hospitalized patients. Old and new drugs costs were compared.

Results: The cost of a 14-day treatment course for methicillin-resistant Staphylococcus aureus bacteremia with a newly approved drug was found to be 6 to 60 times higher than that of older drugs. Similarly, the cost of a 14-day course for carbapenem-resistant Enterobacterales or MDR Pseudomonas aeruginosa was doubled with new drugs; and for carbapenemresistant Acinetobacter baumannii, $\sim 20$ times higher with newer drugs. Annual incremental costs of treating difficult-to-treat Gram-negative bacteria with new drugs ranged from 30 million to over 500 million USD.

Conclusions: Using newly approved antibiotic drugs for MDR infections carries a large incremental cost. Additional data to support survival benefit of these drugs are required to justify the price differences. Subgroups of patients who would benefit most from treatment should be defined.

Keywords: Antibiotics; Costs analysis; Guidelines; MDR 


\section{Key Summary Points}

\section{Why carry out this study?}

Guidelines for treatment of multidrugresistant (MDR) bacteria rely on newly approved antibiotics.

We aimed to assess how much would it cost to base treatment for multidrugresistant bacterial infections on those new antibiotics.

\section{What was learned from the study?}

Annual incremental cost of new antibiotics could reach 30-500 million USD for some bacteria.

Lack of solid evidence for superior effectiveness of new antibiotics for these bacteria complicates treatment decisions.

Cost should be part of the discussion while considering use of newly approved antibiotics until further evidence for effectiveness accumulates.

\section{DIGITAL FEATURES}

This article is published with digital features, including a summary slide, to facilitate understanding of the article. To view digital features for this article go to https://doi.org/10.6084/ m9.figshare.13663976.

\section{INTRODUCTION}

Multidrug-resistant (MDR) bacterial infections are a growing problem worldwide. According to a recent Centers for Disease Control and Prevention (CDC) report, over 2.8 million cases of antibiotic-resistant infections occur annually in the US, resulting in over 35,000 deaths [1]. In addition to the medical challenges posed by these infections, they also constitute a public health and economic burden. The spending for one MDR infection has been reported to be $165 \%$ higher than for non-MDR infection, with an incremental cost of 1383 USD. This has been translated to a national annual cost of 2.2 billion USD in 2014, mostly attributed to antibiotic costs $[2,3]$.

Recently, the Infectious Diseases Society of America (IDSA) issued guidelines for treatment of antimicrobial-resistant Gram-negative bacteria. Newly approved drugs dominate treatment recommendations in these guidelines, although their effectiveness for specific MDR infections is supported by limited evidence for survival benefit $[4,5]$.

We aimed to evaluate the estimated cost of using newly approved antibiotics compared to old antibiotics for the treatment of specific difficult-to-treat pathogens.

\section{METHODS}

We searched the US Food and Drugs Administration (FDA) website for antibacterial drugs with in vitro activity against specific MDR ESKAPE bacteria [vancomycin-resistant Enterococcus faecium (VRE), methicillin-resistant Staphylococcus aureus (MRSA), carbapenemresistant Klebsiella pneumoniae, carbapenem-resistant Acinetobacter, MDR Pseudomonas aeruginosa, and carbapenem-resistant Enterobacter spp.) [6]. ESKAPE pathogens were chosen as bacteria of interest based on World Health Organization definition of these bacteria as critical or high priority for research and drug development [7]. Drugs approved between 1 January 2010 and 14 November 2020 were included, and were considered "new" [6]. For each resistant pathogen, we created a list of potentially covering antibiotics, divided into "old" and "new" according to approval before and after 1 January 2010, respectively $[4,6,8]$. Drugs not approved for use in the US were excluded (teicoplanin, intravenous fosfomycin). For each drug, the cost per day and for a 14-day treatment course were determined using the IBM Micromedex Red Book website [9]. The annual price of treating each bacteria using its individual drug options was calculated by multiplying the cost of a 14-day treatment 


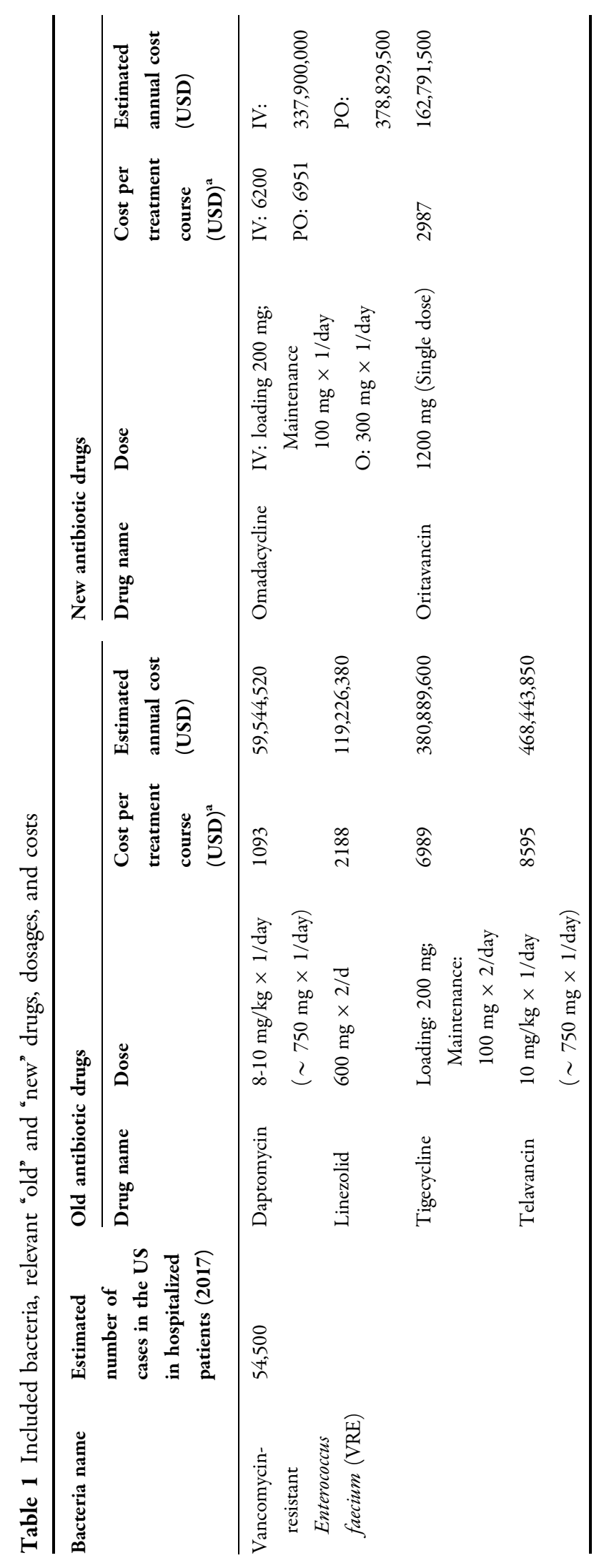




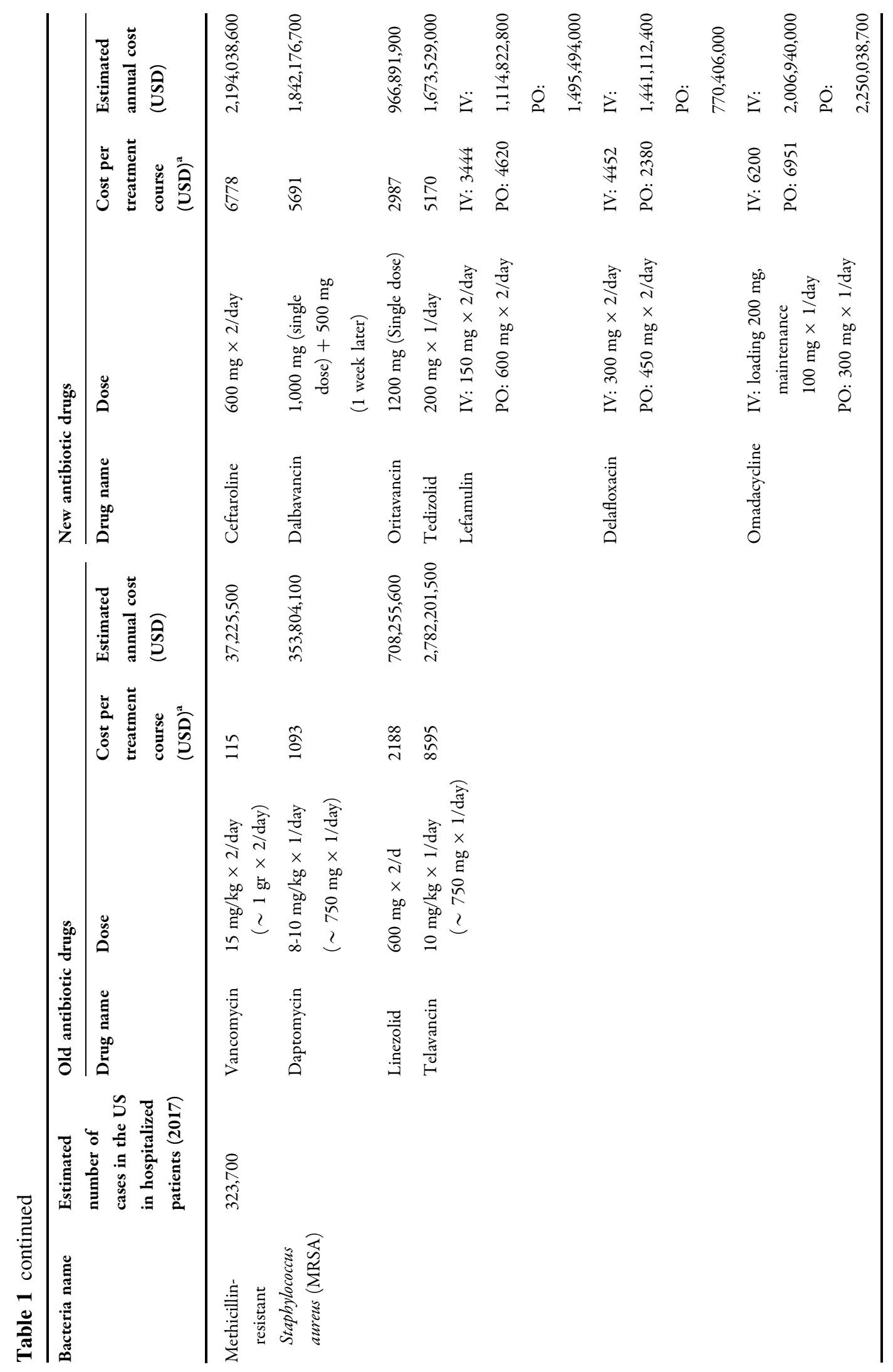




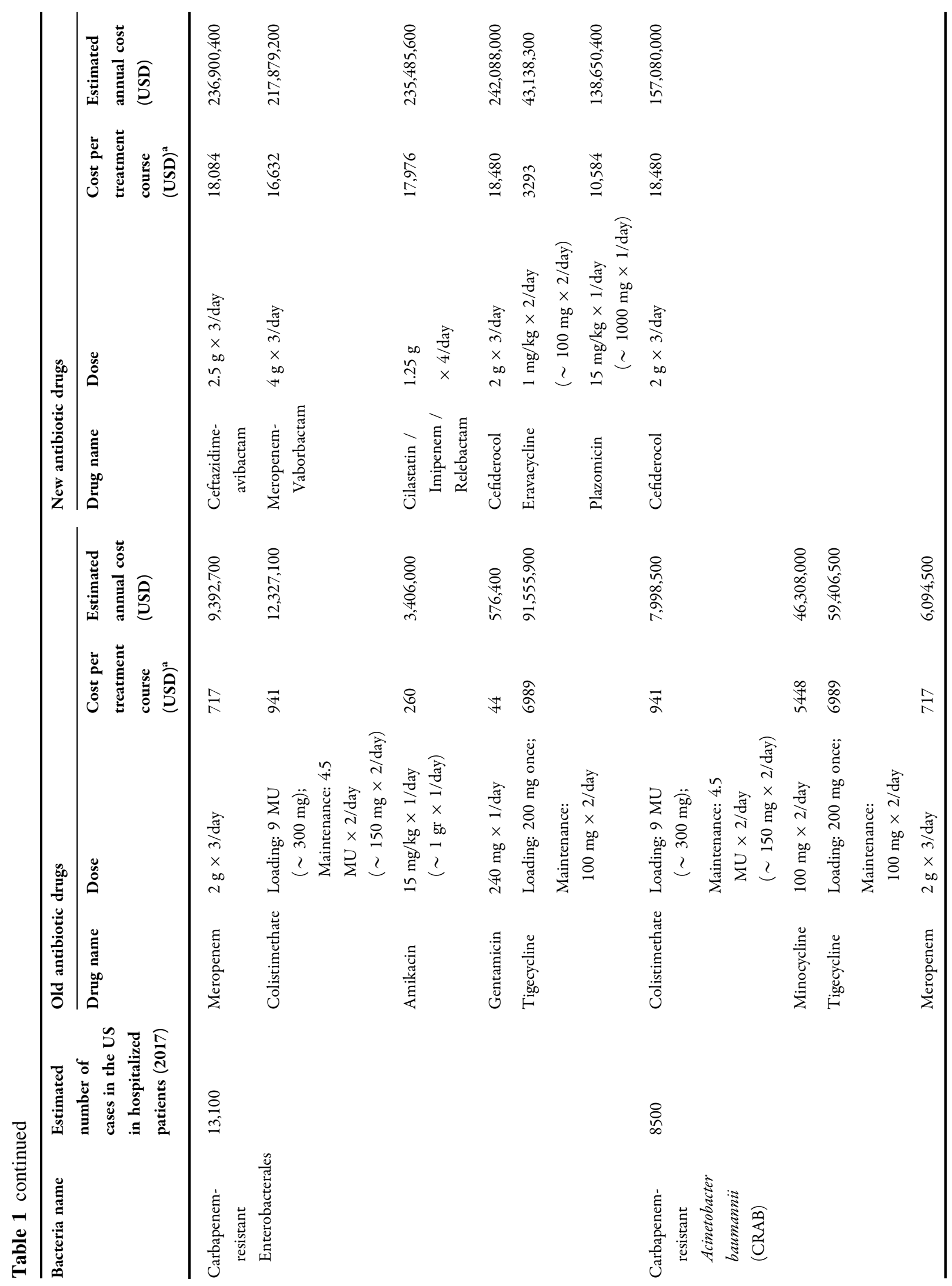




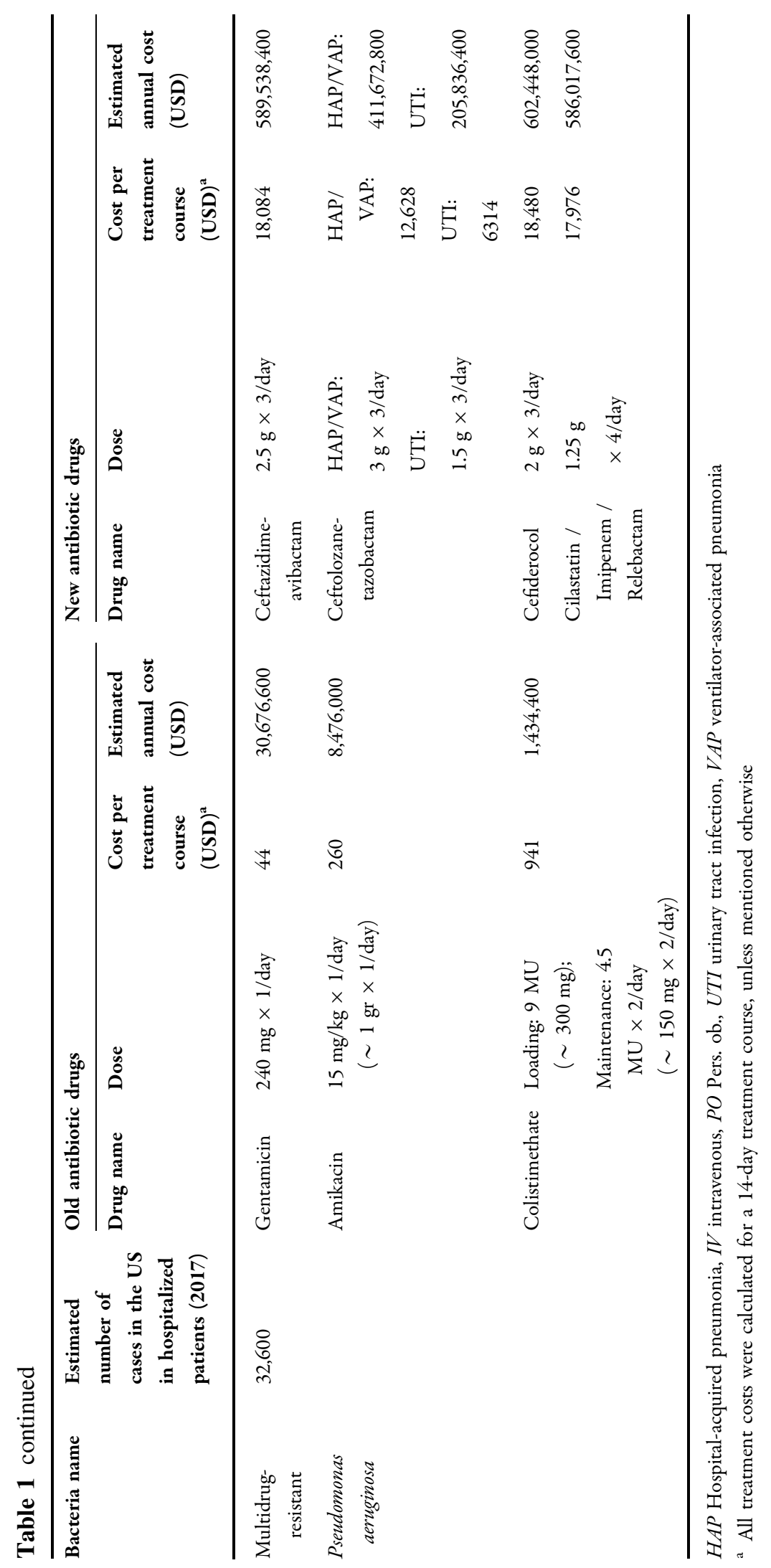


course and the estimated number of annual infections, as detailed in the recent CDC report [1] (see details regarding cost calculation in Supplement 1). Ethical approval was not required for this study, as data were collected from public databases.

\section{RESULTS}

The list of included bacteria, their relevant "old" and "new" drugs, and daily costs are provided in Table 1.

For VRE, the most commonly used old drug cost for a 4-day treatment course ranged between 1093 USD (daptomycin) and 2188 USD (linezolid), and reached up to 6989 USD (tigecycline) and 8595 USD (telavancin). New drug options for VRE are limited, and include omadacycline (6200 USD per course), and oritavancin (2987 USD per course of one dose).

For MRSA bacteremia, a 14-day course of the new drug ceftaroline would cost over six times more than a course of daptomycin (6778 vs. 1093 USD), and $\sim 60$ times the cost of 14 days of vancomycin, the current first-line treatment for these infections [10]. For other sources of infection, the costs of old drugs are also significantly lower for MRSA pneumonia, with 14 days of ceftaroline or lefamulin treatment costing at least 2-3 times more than for linezolid and 40-60 times than for vancomycin, while, for skin and soft tissue infections, clindamycin, trimethoprim-sulfamethoxazole, and doxycycline would have negligible costs compared to lipoglycopeptides, delafloxacin, or omadacycline (Table 1).

For MDR Gram-negative infections, treatment with old drugs is based on various combinations of colistin, tigecycline, meropenem, and aminoglycosides. Recent IDSA guidelines recommend as preferred treatments for carbapenem-resistant Enterobacterales (CRE) and difficult-to-treat Pseudomonas aeruginosa mainly new drugs, including ceftazidime-avibactam, ceftolozane-tazobactam, meropenem-vaborbactam, imipenem-relebactam, and cefiderocol [4]. A treatment course with any of these would cost double that for the colistin-tigecycline combination and $\sim 20$ times the cost of colistin monotherapy, commonly used for Carbapenem-resistant Acinetobacter baumannii (CRAB) infections [11].

Considering 8500 annual CRAB infections in hospitalized patients [1], treating all of them with cefiderocol instead of colistin would have an annual incremental cost of $\sim 150$ million USD, 20 times that of colistin, based on randomized controlled trials (RCT) showing increased mortality with the former drug [12]. Similarly, the additional treatment cost of 3100 annual carbapenem-resistant Enterobacteriaceae infections with ceftaizidime-avibactam over the colistin-tigecycline combination would have an incremental cost of $\sim 31$ million USD, more than doubling the cost. For 32,600 MDR $P$. aeruginosa annual cases, imipenem-relebactam treatment would cost 577 million USD more than amikacin, 69 times the cost of the older drug.

\section{DISCUSSION}

Infections with ESKAPE pathogens are lifethreatening, severe infections, carrying substantial mortality. Preventive strategies have accomplished a reduction/stabilization in the number of ESKAPE infections in the US [1]. Yet, highly resistant Gram-negative infections were recently estimated to require between 39 and 138.2 days of therapy for 10,000 patient encounters. In other countries, rates are even higher, with over $10 \%$ of Gram-negative bacteremias caused by difficult-to-treat resistant pathogens [13].

Due to the severity and poor outcome of these infections, development of new drugs has been prioritized by policy-makers. Four years after FDA's approval of ceftazidime-avibactam, new anti-CRE drugs were reported to be used less widely than expected. Explanations suggested for the relatively low uptake (estimated at $35 \%$ ) include high cost, shortage or nonavailability issues, and lack of evidence from RCTs supporting superior efficacy and safety [14]. Ongoing emergence of resistance to these new drugs could also contribute to the restricted use, as well as delays in the availability of susceptibility testing methods $[13,15]$. Even with 
restricted use, annual sales of ceftazidime-avibactam, meropenem-vaborbactam, and plazomicin in 2018-9 were estimated at 101 million USD, while it has been estimated that, with a $100 \%$ uptake, the cost would have been 289 million [13].

Recent IDSA guidelines for the treatment of difficult-to-treat Gram-negative infections rely mainly on new antibiotics [4]. Recommendations in these guidelines were based on observational studies and two small RCTs, showing mortality benefit of meropenem-vaborbactam for carbapenem-resistant Enterobacterales infections and imipenem-relebactam for MDR Pseudomonas infections [16]. Cefiderocol, as treatment for carbapenem-resistant Gram-negative bacteria, was demonstrated to result in increased all-cause mortality compared with colistin-based therapy in the only RCT published for this indication [12].

We found that the incremental cost of selecting new drugs over older ones could reach hundreds of millions of USD annually, with limited evidence for superior effectiveness. These costs were calculated for hospitalized patients, not considering the increased burden of such infections in other institutional sites of care, such as nursing homes.

Trials addressing new drugs specifically for highly resistant pathogens are scarce, and the evidence for the use of some of these drugs is sometimes very poor. We found that using the ceftaroline for MRSA bacteremia, which has never been tested in an RCT, would cost 60 times the cost of vancomycin. Nevertheless, Gram-positive infections are less of a problem compared to Gram-negative ones. Many options are available for treating skin and soft tissue infections caused by MDR Gram-positive infections, and the choice of using new drugs may be only for the purpose of oral step-down or the use of single-dose administration. For Gram-negative bacteria, clinicians may face infections that are resistant to all older drugs, since resistance to colistin and meropenem have increased, and the use of new drugs would likely be necessary. Regarding Gram-negative infections, the annual cost of cefiderocol for CRAB infections was estimated to be 20 times that of colistin. The only RCT comparing the two showed increased all-cause mortality with cefiderocol [12, 17]. Trials like the latter, specifically including patients with MDR infections, are difficult to conduct. Their performance involves identifying a sufficient number of patients with a possibly life-threatening infection, and obtaining their consent to receive an old drug perceived to be less effective.

It should be noted that our cost analysis is limited to the situation in the US. Hence, the generalization of our data may be limited, and may depend on the cost of the drugs in other countries, the epidemiology of resistant bacteria, and the availability of new drugs.

The discussion regarding antibiotic cost may change in the near future if the UK's innovative 'subscription-type' payment model gets broad acceptance. In this model, the UK's National Health Service will pay a bulk sum for an annual payment to pharmaceutical companies, purchasing the whole yearly supply of necessary antibiotics based on the health benefits to patients according to NHS consideration. This will likely negate the need to specifically consider the price of each antibiotic before treating a patient, and is also thought to be able to secure a constant pipeline of new antimicrobials, by providing companies with an upfront payment which can be used in future development ventures. Two antibiotic drugs that were first selected for purchase in this model are cefiderocol and ceftazidime-avibactam [18].

\section{CONCLUSIONS}

Older drugs have limited effectiveness and some have considerable toxicities [4]. The development of new drugs is of high priority, and their use may provide important benefits for patients, including a survival benefit. Nevertheless, additional proof of such benefits should come from clinical trials, and drug prices should be part of the discussion while considering the use of these drugs. Local protocols regarding antibiotic use should take into account regional costs and the availability of new antibiotics, in addition to the epidemiology of various MDR bacteria. Assessment of the risk of toxicity and/ or reduced effectiveness should be conducted 
on a case-by-case basis prior to decisions on an antibiotic regimen. Subgroups of patients who would benefit most from these new, expensive drugs, should be defined, and include populations often excluded or under-represented in RCTs, e.g., immunocompromised patients, patients with baseline renal dysfunction, and elderly patients. Future studies should include these patients and report results specifically for these subgroups.

\section{ACKNOWLEDGEMENTS}

Funding. No funding or sponsorship was received for this study or publication of this article.

Authorship. All named authors meet the International Committee of Medical Journal Editors (ICMJE) criteria for authorship for this article, take responsibility for the integrity of the work as a whole, and have given their approval for this version to be published.

Authorship Contributions. Study concept was perceived by Noam Tau; study design: Noam Tau, Dafna Yahav; acquisition of data: Noam Tau, interpretation of data: Noam Tau, Dafna Yahav, Daniel Shepshelovich, drafting of the manuscript: Noam Tau, Dafna Yahav; critical revision of the manuscript: all authors.

Disclosures. Dafna Yahav, Daniel Shepshelovich and Noam Tau have nothing to disclose.

Compliance with Ethics Guidelines. Ethical approval was not required for this study, as data were collected from public databases.

Data Availability. Data sharing is not applicable to this article as no datasets were generated or analyzed during the current stud other than the data provided in Table 1.

Open Access. This article is licensed under a Creative Commons Attribution-NonCommercial 4.0 International License, which permits any non-commercial use, sharing, adaptation, distribution and reproduction in any medium or format, as long as you give appropriate credit to the original author(s) and the source, provide a link to the Creative Commons licence, and indicate if changes were made. The images or other third party material in this article are included in the article's Creative Commons licence, unless indicated otherwise in a credit line to the material. If material is not included in the article's Creative Commons licence and your intended use is not permitted by statutory regulation or exceeds the permitted use, you will need to obtain permission directly from the copyright holder. To view a copy of this licence, visit http://creativecommons.org/licenses/by$\mathrm{nc} / 4.0 /$.

\section{REFERENCES}

1. CDC (Centers for Disease Control and Prevention). ANTIBIOTIC RESISTANCE THREATS IN THE UNITED STATES 2019. 2019. https://www.cdc.gov/ drugresistance/pdf/threats-report/2019-ar-threatsreport-508.pdf. Accessed 1 Dec 2020.

2. Thorpe KE, Joski P, Johnston KJ. Antibiotic-resistant infection treatment costs have doubled since 2002, now exceeding USD2 billion annually. Health Aff Proj Hope. 2018;37(4):662-9. https://doi.org/10. 1377/hlthaff.2017.1153.

3. Serra-Burriel M, Keys M, Campillo-Artero C, et al. Impact of multi-drug resistant bacteria on economic and clinical outcomes of healthcare-associated infections in adults: Systematic review and meta-analysis. PLoS ONE. 2020;15:1. https://doi. org/10.1371/journal.pone.0227139.

4. Tamma PD, Aitken SL, Bonomo RA, Mathers AJ, van Duin D, Clancy CJ. Infectious diseases society of america antimicrobial resistant treatment guidance: gram-negative bacterial infections. Clin Infect Dis. 2020. https://doi.org/10.1093/cid/ciaa1478.

5. Yahav D, Tau N, Shepshelovich D. Assessment of data supporting the efficacy of new antibiotics for treating infections caused by multidrug-resistant bacteria. Clin Infect Dis. 2020. https://doi.org/10. 1093/cid/ciaa457.

6. Drugs@FDA: FDA Approved Drug Products. 2019. https://www.accessdata.fda.gov/scripts/cder/daf/. Accessed 12 Jul 2019. 
7. Tacconelli E, Carrara E, Savoldi A, et al. Discovery, research, and development of new antibiotics: the WHO priority list of antibiotic-resistant bacteria and tuberculosis. Lancet Infect Dis. 2018;18(3): 318-27. 3099(17)30753-3.

8. Theuretzbacher U, Bush K, Harbarth S, et al. Critical analysis of antibacterial agents in clinical development. Nat Rev Microbiol. 2020;18(5):286-98. https://doi.org/10.1038/s41579-020-0340-0.

9. IBM. IBM Micromedex RED BOOK. 2020. https:// www.ibm.com/products/micromedex-red-book. Accessed 1 Dec 2020.

10. Liu C, Bayer A, Cosgrove SE, et al. Clinical practice guidelines by the infectious diseases society of america for the treatment of methicillin-resistant Staphylococcus aureus infections in adults and children. Clin Infect Dis. 2011;52(3):e18-55. https:// doi.org/10.1093/cid/ciq146.

11. Paul M, Daikos GL, Durante-Mangoni E, et al. Colistin alone versus colistin plus meropenem for treatment of severe infections caused by carbapenem-resistant Gram-negative bacteria: an open-label, randomised controlled trial. Lancet Infect Dis. 2018;18(4):391-400. https://doi.org/10. 1016/S1473-3099(18)30099-9.

12. Bassetti M, Echols R, Matsunaga Y, et al. Efficacy and safety of cefiderocol or best available therapy for the treatment of serious infections caused by carbapenem-resistant Gram-negative bacteria (CREDIBLE-CR): a randomised, open-label, multicentre, pathogen-focused, descriptive, phase 3 trial.
Lancet Infect Dis. 2020. https://doi.org/10.1016/ S1473-3099(20)30796-9.

13. Strich JR, Warner S, Lai YL, et al. Needs assessment for novel Gram-negative antibiotics in US hospitals: a retrospective cohort study. Lancet Infect Dis. 2020;20(10):1172-81. https://doi.org/10.1016/ S1473-3099(20)30153-5.

14. Clancy CJ, Potoski BA, Buehrle D, Nguyen MH. Estimating the treatment of carbapenem-resistant enterobacteriaceae infections in the United States using antibiotic prescription data. Open Forum Infect Dis. 2019;6(8):344. https://doi.org/10.1093/ ofid/ofz344.

15. Satlin MJ. Languid uptake of ceftazidime-avibactam for carbapenem-resistant gram-negative infections and continued reliance on polymyxins. Clin Infect Dis. 2020. https://doi.org/10.1093/cid/ciaa065.

16. Yahav D, Giske CG, Grāmatniece A, Abodakpi H, Tam VH, Leibovici L. New $\beta$-Lactam- $\beta$-lactamase inhibitor combinations. Clin Microbiol Rev. 2020;34:1. https://doi.org/10.1128/CMR.00115-20.

17. Powers Iii JH. Scientific evidence, regulatory decision making and incentives for therapeutics in infectious diseases: the example of cefiderocol. Clin Infect Dis. 2020. https://doi.org/10.1093/cid/ ciaa1795.

18. Robinson, J. First antimicrobial drugs purchased via new 'subscription' payment model. Pharmaceut J. 2021;306:7945. 